\title{
Changes of Bcl-2, Bax and Caspase-3 expression in the dermal microvascular endothelial cells and the epidermal layers of the eschar (tache noire) in patients with Mediterranean spotted fever
}

\author{
Ivan Baltadzhiev', Slavi Delchev² \\ ${ }^{1}$ Department of Infectious Diseases, Parasitology and Tropical Medicine, Medical University, \\ Plovdiv, Bulgaria \\ ${ }^{2}$ Department of Anatomy, Histology and Embryology, Medical University, Plovdiv, Bulgaria
}

\begin{abstract}
Mediterranean spotted fever (MSF) is widely prevalent in many endemic regions in Bulgaria. The disease is still not quite thoroughly studied as to some aspects of its pathogenesis and especially to issues that concern the crucial signals for apoptosis in the target microvascular endothelial cells. To study the expression of Bcl-2 family proteins and Caspase- 3 in the dermal capillary endothelial cells from skin papules and in the eschar (tache noire) epidermal layers of patients with MSF so that we can establish apoptotic processes and the time of their occurrence and deployment. Immunohistochemical reactions for Bcl-2, Bax and Caspase-3 were obtained in slices of punch-biopsies taken from papules of the skin rash and from the eschars of eight patients with MSF. The average intensity of the reactions was compared with that in control punch-biopsy slices from four healthy subjects. MSF was etiologically confirmed in all patients by positive antibody response to a specific antigen, Rickettsia conorii, with indirect immunofluorescent assay performed by the Rickettsial Reference Laboratory. The immune reaction for Bcl-2 was found to be poorly expressed in the capillary endothelial cells of skin papules of patients without any differences from controls. The expression of Bax and Caspase-3 was strongly upregulated in comparison with the controls. The Bcl-2/Bax ratio was significantly decreased. Microvascular endothelial cells of the eschar showed similar changes. While the Bcl-2/Bax ratio decreased in the epidermal layers of the eschar "tache noire", there were no changes in the intensity of the immunoreactivity of Caspase- 3 as compared with controls. The upregulation of Bax and Caspase- 3 is an indication of ongoing apoptotic processes in the dermal microvascular endothelial cells of MSF patients. The epidermal layers of the eschar showed increased sensitivity to apoptosis, however, executive phase of apoptosis did not occur. (Folia Histochemica et Cytobiologica 2013, Vol. 51, No. 2, 121-126)
\end{abstract}

Key words: mediterranean spotted fever, Rickettsia conorii, endothelial cells, eschar, apoptosis, Bcl-2, Bax, caspase-3, immunohistochemistry

\section{Introduction}

Mediterranean spotted fever (MSF) caused by Rickettsia conorii conorii transmitted by the brown dog

\footnotetext{
Correspondence address: I. Baltadzhiev,

Department of Infectious Diseases,

Parasitology and Tropical Medicine,

Medical University of Plovdiv,

15A Vassil Aprilov Blvd., 4002 Plovdiv, Bulgaria

tel./fax: +35932268341/+35932602851

e-mail: ivan_balt@yahoo.com
}

tick Rhipicephalus sanguineus has been widely prevalent in many endemic regions in Bulgaria for the last 20 years $[1,2]$. Rickettsiae are obligate intracellular parasites with prominent tropism for the host vascular endothelial cells $[3,4]$. The rickettsial diseases are still not quite thoroughly studied as to some aspects of their pathogenesis and especially to issues that concern the crucial signals for apoptosis in the targeted microvascular endothelial cells [4-6].

To survive, rickettsiae are capable of either inducing or inhibiting apoptosis during different stages of infection $[4,7-8]$. The programmed cell death in MSF is among the 
phenomena that are still inadequately elucidated $[4-6,9]$. We were prompted to conduct the present study by the scantiness of studies on apoptosis in the microvascular endothelial cells in rickettsial diseases and by practically nonexistent research on patients with MSF [10-13]. This study is the first in this field in Bulgaria and we have found no similar studies available in the pertinent literature. We aimed at studying the expression of Bcl-2 family proteins and Caspase- 3 in the dermal capillary endothelial cells from skin papules, as well as in the epidermal layers from the eschar (tache noire) of patients with MSF in order to find out if apoptotic processes occur there, when they occur and how they are deployed.

\section{Material and methods}

Rickettsia conorii was confirmed as etiological agent by immunofluorescence assay (IFA) in the Rickettsial Reference Laboratory of the Military Medical Academy in Sofia. The subject of the study was approved by the Ethics Committee at the Medical University - Plovdiv. Written informed consent was obtained from all participants in the study.

We examined punch biopsies from skin papules and from the inflammatory infiltrate of the eschar taken off the central necrotic area in eight patients with MSF ( 4 men and 4 women, aged 28 to 66 years). Punch biopsies taken from matched skin areas of 4 healthy subjects served as controls. The following selection criteria to the patients enrolled in the study were applied:

Inclusion criteria:

- presence of tache noire and febrile illness with flu-like symptoms for 3-4 days prior to the onset of typical maculopapular rash in a patient from an endemic for MSF area in a suitable for the disease season; Four fold rise of R.conorii antibody titer in double serum samples;

- first patients' presentation to the hospital 48 hours after the rash onset;

- lack of antibiotic treatment before the biopsy performance.

Exclusion criteria:

- patients (control subjects) with underlying chronic diseases (chronic renal failure, diabetes mellitus, ischemic heart disease, malignancy, trauma, infections and rheumatic disease, etc.); patients (control subjects) with pre-existing or concurrent skin disorders;

- whatever treatment except antipyretics before hospitalization and biopsy;

- children (ethical considerations).

The biopsy specimens were processed by standard methods and embedded into paraffin. Consecutive $5 \mu \mathrm{m}$ thick slices were prepared, mounted on silane-covered slides and examined immunohistochemically. We used a mouse ABC staining system (sc-2017, kit, Santa Cruz Biotechnology, Dallas, TX, USA) and the following primary antibodies in a 1:100 dilution: monoclonal antibody Bcl-2 (sc-509, Santa Cruz Biotechnology); monoclonal antibody Bax (sc-7480, Santa Cruz Biotechnology); monoclonal antibody Caspase-3 (sc-56046, Santa Cruz Biotechnology). Additionally, the specimens were stained with hematoxylin. The prepared slices were covered with Vector Mount (Vector Lab, Burlingame, CA, USA). The microphotographic pictures were taken by a Nikon Microphot-SA (Tokyo, Japan) microscope, equipped with a Camedia-5050Z digital camera (Olympus, Tokyo, Japan). The expression of the immune reaction for Bcl-2, Bax and Caspase-3 in the dermal vascular endothelial cells and epidermal layers was recorded using specialized software 'DP - Soft' 3.2 (Olympus,). The average intensity of the reaction of 50 randomly selected areas in each cross-section was analyzed. The values for the intensity of immunochemical reactions for Bcl-2, Bax and Caspase-3 are presented in relative units. The results (mean $\pm \mathrm{SD}$ ) were analyzed with the independent samples $t$-test and $\mathrm{p}<0.05$ was considered significant.

\section{Results}

We investigated the expression of the immune reaction for Bcl-2, Bax and Caspase-3 in tissues from skin papules of patients admitted to the hospital 48 hours after the onset of rash - a term we considered as possibly the earliest for the apoptotic processes occurrence in the skin microvascular endothelial cells. Patients' treatment started immediately after the biopsy specimens were obtained. Simultaneously, biopsies were taken from the tache noire. Visually the immune reactions for $\mathrm{Bcl}-2$, Bax and Caspase- 3 in the capillary endothelial cells were manifested by mild-brownish to no staining for Bcl-2 and dark-brown to black coloring for Bax and Caspase- 3 which corresponded to the data of other authors $[14,15]$ (Figure 1). There was a weak expression of the immune reaction for Bcl-2 in the capillary endothelial cells of patient skin papules, and a similar weak expression in the controls $(p>0.05)$. The expression of the immune reaction for Bax was strongly manifested in comparison with the controls $(p<0.001)$. The analysis revealed increased intensity of the immunohistochemical reaction for Caspase-3 in the microvascular endothelium in comparison with the controls $(\mathrm{p}<0.001)$ (Figure 2). The ratio of Bcl-2 to Bax was found to be significantly decreased in patients $(\mathrm{p}<0.001)$ mainly due to the increased staining intensity of the pro-apoptotic protein Bax. Similar to the alterations observed in the capillary endothelial cells of skin papules were found in the microvascular endothelium of the eschar (tache noire).

The analysis of the immune reaction in the epidermal layers of the patients' eschar "tache noire" showed weakened immunostaining for Bcl-2 in keratinocytes of the basal and spinous epidermal layers in comparison with the controls $(\mathrm{p}<0.01)$. Immunoexpression of Bax was increased in the cells of the spinous and 

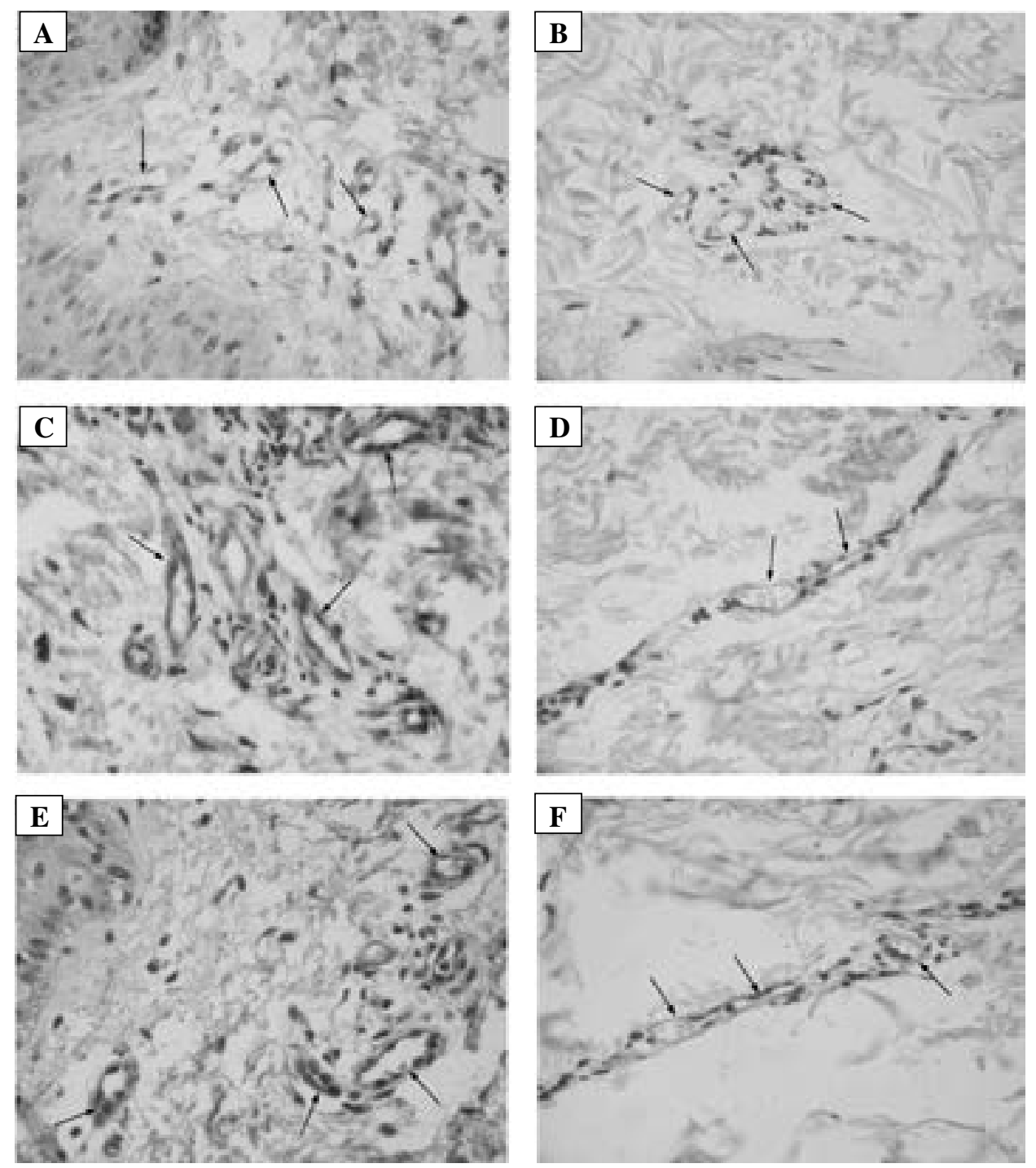

Figure 1. Immunohistochemical reactions for Bcl-2 (A, B), Bax $(\mathbf{C}, \mathbf{D})$ and Caspase-3 $(\mathbf{E}, \mathbf{F})$ in the dermis of skin papules of patients with MSF (A, C, E) and control healthy subjects $(\mathbf{B}, \mathbf{D}, \mathbf{F})$. Arrows - reaction in the dermal capillary endothelial cells. Original magnification $\times 400$

granular layers compared with the controls $(\mathrm{p}<0.05)$. Intensity of the reaction for Caspase- 3 in all layers did not show statistically significant changes in patients compared with the controls (Figures 3 and 4).

\section{Discussion}

One of the diagnostic features of MSF is the characteristic papular (boutonneuse) skin rash. There are capillary and lymphatic networks, and nerve endings in the superficial papillary layer of the connective part of the skin, the dermis [16]. As we searched for signs of apoptosis in the microvascular endothelial cells we obtained the biopsy from the patients' rash papules as these reflect the disrupted capillary permeability, exudation and focal cell infiltration caused by the intracellular pathogen $R$. conorii. In the regulation of programmed host-cell death, the B-cell lymphoma-2
(Bcl-2) family of proteins, which includes both pro- and anti-apoptotic factors, plays a crucial role by controlling the mitochondrial permeability [17-19]. The anti-apoptotic proteins $\mathrm{Bcl}-2$ and $\mathrm{Bcl}-\mathrm{xL}$ reside in the outer membrane of mitochondria; they inhibit the release of cytochrome C [20]. In contrast, the pro-apoptotic proteins Bad, Bid and Bax are located in the cytosol and their translocation to mitochondria causes the release of cytochrome $\mathrm{C}$ [21]. Determination of the effects of Bcl-2 family on nuclear factor NF-kB reveals significant changes in the expression of various pro-and anti-apoptotic proteins which ultimately results in the so called 'equilibrium shift' toward apoptosis [22]. It is in this way that the concept of regulation of the mechanisms of programmed death of endothelial cells by intracellular rickettsiae was proposed [11]. Inhibition of apoptosis through activation of nuclear factor NF-kB is required for the early phase of the disease when rickettsiae 


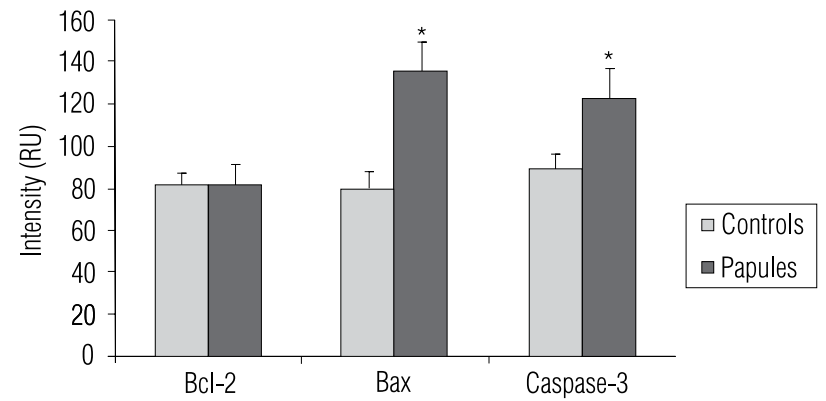

Figure 2. Intensity of the immunoreactivity of Bcl-2, Bax and Caspase-3b expression in the capillary endothelial cells of the skin papules of MSF patients and control subjects. Values represent means \pm SD obtained in the way described in Material and methods. * $p<0,001$ vs. control. RU — relative units. Only statistically significant differences are shown begin to proliferate in the microvascular endothelial cells [13,22-24]. Later, when adaptive immunity is fully deployed apoptosis is enhanced in infected endothelial cells [25].

We investigated the expression of the immune reaction for Bcl-2, Bax and Caspase-3 in tissues from skin papules of patients who were hospitalized two days after the onset of the rash. We considered two days after the rash onset (which is 5-6 days after the initial occurrence of the symptoms of MSF) to be sufficient for the cell-mediated immune response to develop and for anti-apoptotic activity of $R$. conorii to get affected. Therefore we searched for the elements of apoptosis in the endothelial cells of the dermal microvessels using immunohistochemical reactions for the anti-apoptotic protein $\mathrm{Bcl}-2$ and the pro-apoptotic proteins Bax and Caspase-3. Bax is an essential
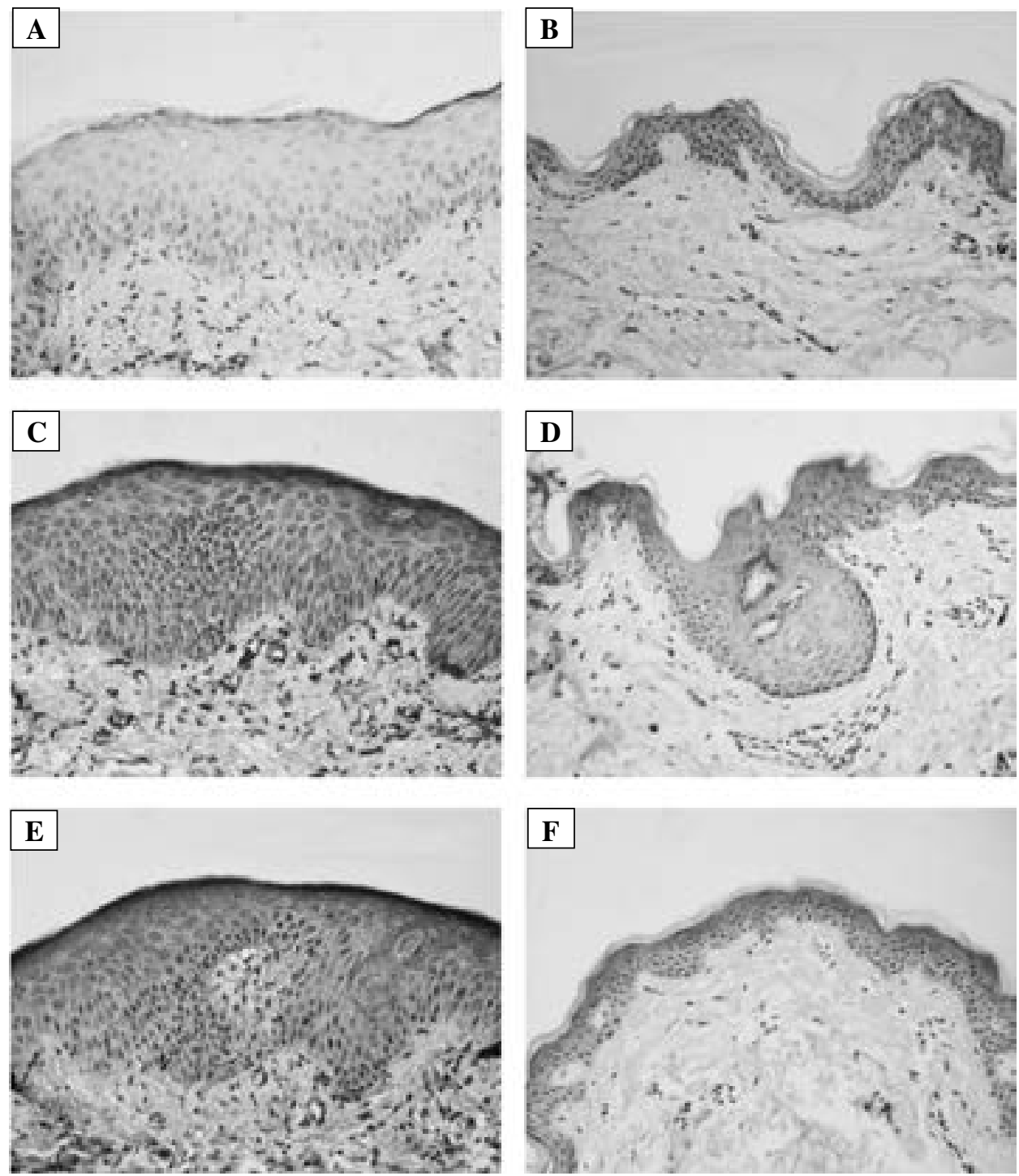

Figure 3. Immunohistochemical reactions for Bcl-2 (A, B), Bax (C, D) and Caspase-3 (E, F) in the epidermis of the eschar 'tache noire' of patients with MSF (A, C, E) and controls of healthy subjects $(\mathbf{B}, \mathbf{D}, \mathbf{F})$. Original magnification $\times 200$ 


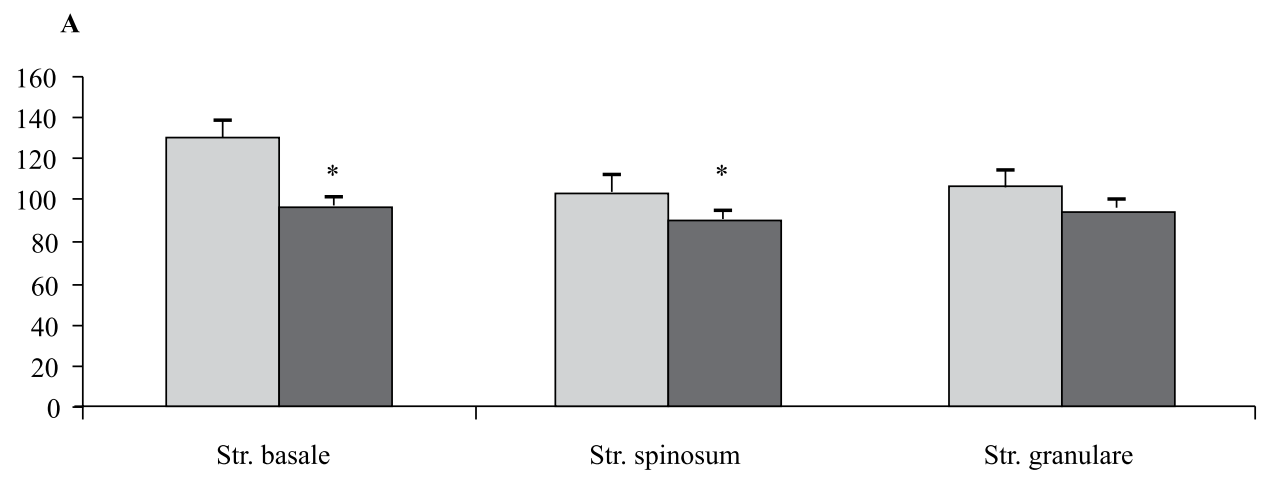

B
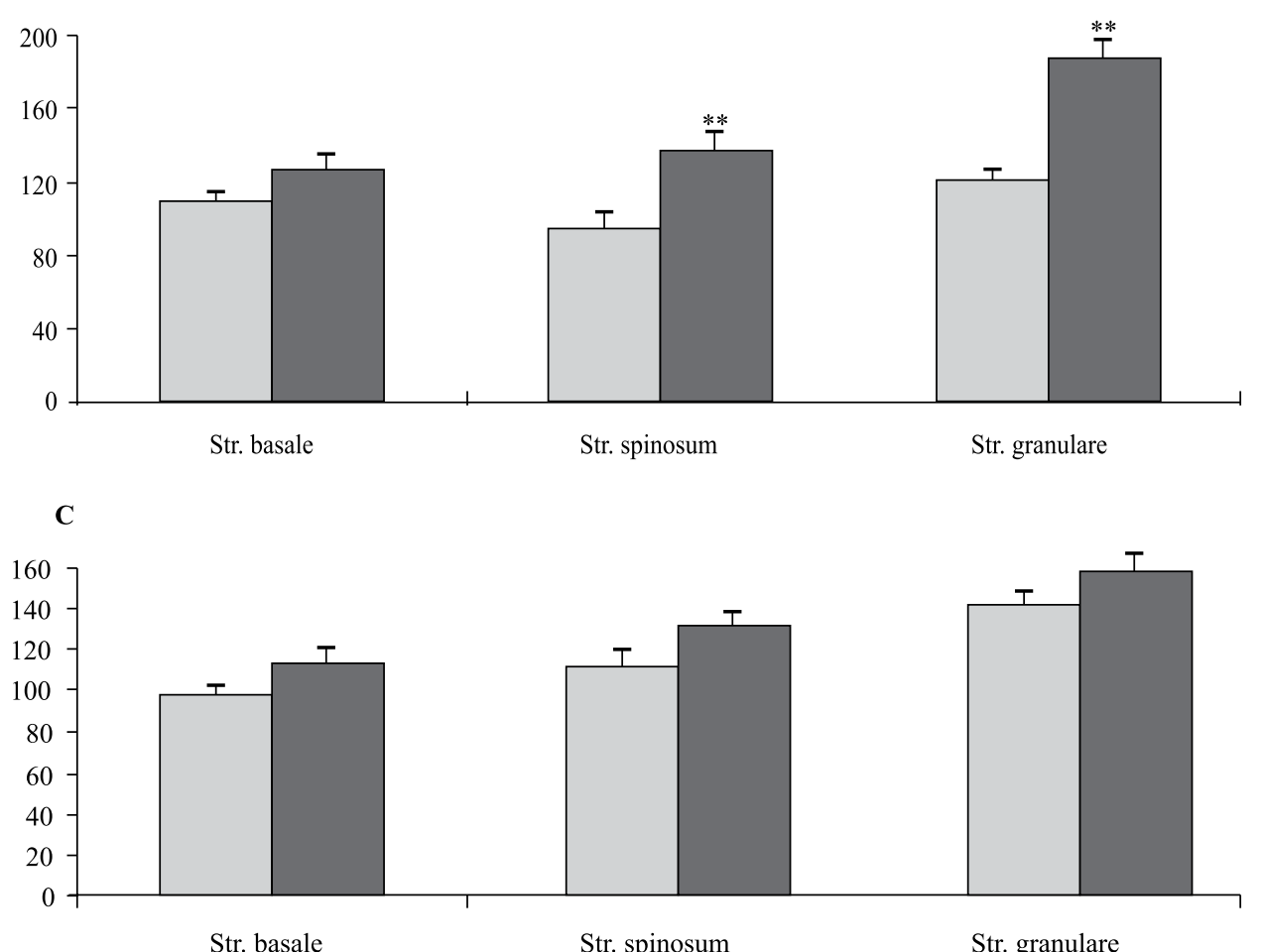

Figure 4. Intensity of the immunoreactivity (mean \pm SD) of Bcl-2, Bax and Caspase-3 expression in the epidermal layers of the eschar ,tache noire” of patients with MSF (shaded bars) and control healthy subjects (open bars). A. Bcl-2, ${ }^{*} \mathrm{p}<0,01 ; \mathbf{B}$. Bax, ${ }^{* *} \mathrm{p}<0,05 ;$ C. Caspase-3. Only statistically significant differences are shown

factor for mitochondria-mediated apoptosis [26-27]. The decreased Bcl-2/Bax ratio in the dermal capillary endothelial cells of skin papules of MSF patients was related to the increased levels (immunoreactivity) of the pro-apoptotic protein Bax which is an indicator of intensified apoptotic sensitivity and initiation of processes of programmed host-cell death.

While Bcl-2 inhibits apoptosis and Bax promotes it, Caspase- 3 is known to be an executioner enzyme in the apoptotic process [28]. The activation of this executioner protease triggers a cascade of proteolytic processes that leads to digestion of structural proteins in the cytoplasm, degradation of chromosomal DNA of the cell and phagocytosis [29]. The observed increased intensity of immunochemical reaction for Caspase-3 in the capillary endothelial cells of the rash papules indicates activation of apoptotic processes and signals early transition to the next stage of the apoptotic cascade that leads to cell death. In light of these considerations, the studied apoptosis markers of microvascular endothelium should attest to the disease process with early or full deployment of T-cell mediated immune response [25]. However, no altered expression of Caspase- 3 in the three epidermal layers of the eschar was found as compared with controls. Thus, the epidermis of the eschar (tache noire) shows increased susceptibility to apoptosis, but the actual process of apoptosis does not take place. These facts suggest that the mechanisms of apoptosis induced in the epidermis as a consequence of rickettsial infection are not identical to the mechanisms triggering apoptosis processes in vascular endothelium. 
In conclusion, determining the time of initiation of the apoptotic processes in the microvascular endothelium of patients is important to detect early cell-mediated processes in the body's defence against the intracellular pathogen $-R$. conorii. The delay of apoptotic processes could indicate a failure of the immune system, or a more severe course of the disease, or the occurrence of complications.

\section{Acknowledgement}

This work was supported by the Research Department of the Medical University - Plovdiv, grant no. HO 8/2008.

We thank Professor N. Popivanova (Medical University of Plovdiv) for helpful discussions.

\section{References}

1. Alexandrov E, Kazar J, Hechemy K, Kantardjiev T, eds. Contemporary state of the rickettsioses in the world and in Bulgaria. Sofia: Prof. Marin Drinov Acad. Publish. House; 2007.

2. Popivanova N, Baltadzhiev 1, Zaprianov Z. Mediterranean spotted fever in the Plovdiv region of Bulgaria. In: Alexandrov E, Kazar J, Hechemy K, Kantardjiev T, eds. Contemporary state of the rickettsioses in the world and in Bulgaria. Sofia: Prof. Marin Drinov Acad. Publish. House; 2007:116-130.

3. Walker DH, Ismail N. Emerging and re-emerging rickettsioses: endothelial cell infection and early disease events. Nat Rev Microbiol. 2008;6:375-386.

4. Sahni S, Rydkina E. Host-cell interactions with pathogenic Rickettsia species. Future Microbiol. 2009;4:323-339.

5. Walker DH. Rickettsiae and rickettsial infections: the current state of knowledge. Clin Infect Dis. 2007;45 (Suppl.1):S39-44.

6. Mansueto P, Vitale G, Cascio A et al. New insight into immunity and immunopathology of Rickettsial diseases. Clin Dev Immunol. 2012;2012:967852.

7. Faherty C., Maureli A. Staying alive: bacterial inhibition of apoptosis during infection. Trends Microbiol. 2008;16:173-180.

8. Bortner CD, Cidlowski JA. Cellular mechanisms for the repression of apoptosis. Annu Rev Pharmacol Toxicol. 2002;42:259-281.

9. Walker DH, Valbuena GA, Olano JP. Pathogenic mechanisms of diseases caused by Rickettsia. Ann N Y Acad Sci. 2003;990:1-11.

10. Joshi SG, Francis CW, Silvermann DJ, Sahni SK. NF-kB activation suppresses host cell apoptosis during Rickettsia rickettsii infection via regulatory effects on intracellular localization or levels of apoptogenic and anti-apoptotic proteins. EMS Microbiol Lett. 2004;234:333-341.

11. Bechelli JB, Rydkina E, Colonne PM, Sahni SK. Rickettsia rickettsii infection protects human microvascular endothelial cells against staurosporine-induced apoptosis by a cIAP2-independent mechanisms. J Infect Dis. 2009;199:1389-1398.

12. Radulovic S, Price PW, Beier MS, Gaywee J, Macaluso JA, Azad A. Rickettsia-macrophage interactions: host cell responses to Rickettsia akari and Rickettsia typhi. Infect Immun. 2002;70:2576-2582.
13. Clifton DR, Goss RA, Sahni SK et al. NF-kB-dependent inhibition of apoptosis is essential for host cell survival during Rickettsia rickettsii infection. Proc Natl Acad Sci USA 1998;95:4646-4651.

14. Hsia JY, Chen CY, Hsu CP, et al. Expression of apoptosis-regulating proteins $\mathrm{p} 53, \mathrm{Bcl}-2$, and Bax in primary resected esophageal squamous cell carcinoma. Neoplasma. 2001;48:483-488.

15. de Haas ER, de Bruijn HS, Sterenborg HJ, Neumann HA, Robinson DJ. Apoptosis markers in ALA-PDT treated superficial basal cell carcinomas using a single and a two fold illumination scheme. In: Ellen RM de Haas: $A L A-P D T$; the treatment of non melanoma skin-cancer re-illuminated. Thesis: Erasmus University, Rotterdam 2007:89-107.

16. Vassilev V, Baltadjiev G, Baltadjiev A. Human Anatomy. Plovdiv: IK-VAP Publish. House; 2009.

17. Gross A, McDonnell JM, Korsmeyer SJ. BCL-2 family members and the mitochondria in apoptosis. Genes Dev. 1999;13:1899-1911.

18. Harris $\mathrm{MH}$, Thompson CB. The role of the Bcl-2 family in the regulation of outer mitochondrial membrane permeability. Cell Death Differ. 2000;7:1182-1191.

19. Kroemer G. The mitochondrion as an integrator/coordinator of cell death pathways. Cell Death Differ.1998;5:547.

20. Scorrano L, Korsmeyer SJ. Mechanisms of cytochrome c release by proapoptotic Bcl-2 family members. Biochem Biophys Res Commun , 2003;304:437-444.

21. Smaili SS, Hsu YT, Carvalho AC, Rosenstock TR, Sharpe JC, Youle RJ. Mitochondria, calcium and pro-apoptotic proteins as mediators in cell death signaling. Braz J Med Biol Res. 2003;36:183-190.

22. Joshi SG, Francis CW, Silverman DJ, Sahni SK. Nuclear factor kappa B protects against host cell apoptosis during Rickettsia rickettsii infection by inhibiting activation of apical and effector caspases and maintaining mitochondrial integrity. Infect Immun. 2003;71:4127-4136.

23. Clifton DR, Rydkina E. Freeman RS, Sahni SK. NF $-\mathrm{kB}$ activation during Rickettsia rickettsii infection of endothelial cells involves the activation of catalytic IkB kinases IKK $\alpha$ and IKK $\beta$ and phosphorilation-proteolysis of the inhibitor protein $\operatorname{IkB} \alpha$. Infect Immun. 2005;73:155-165.

24. Clifton DR, Rydkina E, Huyck H, et al. Expression and secretion of chemotactic cytokines IL-8 and MCP-1 by human endothelial cells after R.rickettsii infection: regulation by nuclear transcription factor NF-kB. Int J Med Microbiol 2005;295:267-278.

25. Walker DH, Olano JP, Feng HM. Critical role of cytotoxic T lymphocytes in immune clearance of rickettsial infection. Infect Immun. 2001;69:1841-1846.

26. Chiu SM, Xue LY, Usuda J, Azizuddin K, Oleinick NL. Bax is essential for mitochondrion-mediated apoptosis but not for cell death caused by photodynamic therapy. Br J Cancer. 2003;89:1590-1597.

27. Finucane DM, Bossy-Wetzel E, Waterhouse NJ, Cotter TG, Green DR. Bax-induced caspase activation and apoptosis via cytochrome c release from mitochondria is inhibitable by Bcl-xL. J Biol Chem. 1999;274:2225-2233.

28. Mazumder S, Plesca D, Almasan A. Caspase-3 activation is a critical determinant of genotoxic stress-induced apoptosis. Methods Mol Biol. 2007;414:13-22.

29. Riedl SJ, Shi Y. Molecular mechanisms of caspase regulation during apoptosis. Nat Rev Mol Cell Biol. 2004;5:897-907.

Submitted: 5 December, 2012

Accepted after reviews: 13 June, 2013 